\title{
Drug output from nebulizers is dependent on the method of measurement
}

\author{
P.W. Barry, C. O'Callaghan
}

Drug output from nebulizers is dependent on the method of measurement. P.W. Barry, C. O'Callaghan. OERS Journals Ltd 1998.

ABSTRACT: The objective of this study was to determine whether current regulatory methods for assessing the output of nebulizers are appropriate for the delivery of nebulized steroid suspensions to patients.

We studied a conventional jet nebulizer (the Intersurgical Cirrus), an open-vent nebulizer (the Medicaid Sidestream) and a breath-enhanced nebulizer (the Pari LC Plus), using a constant sampling flow or a sinusoidal pump to represent the breathing pattern of children from 6 months to adulthood.

Recovery of budesonide released from the nebulizers onto filters was reduced when using breathing simulation compared with constant flow, and this reduction was greatest for the conventional nebulizer (Cirrus, $103 \mu \mathrm{g}$ with constant flow to $4.4 \mu \mathrm{g}$ with a $50 \mathrm{~mL}$ tidal volume; Pari, $176 \mu \mathrm{g}$ to $25 \mu \mathrm{g}$ ). The open-vent nebulizer deposited very little budesonide on the filter at lower tidal volumes $(4.5 \mu \mathrm{g}$ with a $50 \mathrm{~mL}$ tidal volume), possibly because the enhanced flow of aerosol laden air was greater than the inspiratory flow from the breathing simulator. The output of the $\mathrm{LC}$ plus was reduced at high flow, from $176 \mu \mathrm{g}$ at $20 \mathrm{~L} \cdot \mathrm{min}^{-1}$ to $93 \mu \mathrm{g}$ at $60 \mathrm{~L} \cdot \mathrm{min}^{-1}$. Overall, the measured output varied by up to $700 \%$, depending on the method used.

These results suggest that breathing patterns dramatically alter the measured output of different nebulizers and that breathing simulation should be included as part of their assessment.

Eur Respir J 1998; 12: 463-466.
Dept of Child Health, University of Leicester, Leicester, UK.

Correspondence: P.W. Barry

Department of Child Health

University of Leicester

Clinical Sciences Building

Leicester Royal Infirrnary

P.O. Box 65

Leicester LE2 7LX

UK

Fax: 441162523282

Keywords: Drug administration drug inhalation

nebulizer

respiratory therapy

vapourizer

Received: November 51997

Accepted after revision March 131998
Jet nebulizers are popular devices used to deliver drugs to the respiratory tract. Many different devices are available and nebulizer manufacturers typically quote the amount of aerosol produced by their nebulizer in particles smaller than a certain size as an indication of the nebulizer's efficiency. However, current methodologies for assessing nebulizer output outlined in British [1] and proposed European guidelines do not take into account the breathing pattern of the patient. This may be very important for children, who may have tidal volumes smaller than the output of aerosol laden air from the nebulizer, so that they lose aerosol during both inspiration and expiration [2], and for the assessment of new "breath-enhanced" nebulizers, which have been shown to double drug delivery [3], but where assessment at a constant flow may hide important differences in drug output.

In addition, guidelines for nebulizer assessment do not distinguish between different types of medication. Steroid preparations currently available for nebulization are formulated as suspensions and results from experiments using drug solutions cannot necessarily be extrapolated to include them.

To determine whether the method of assessment affects the measured amount of drug delivered, the output of budesonide from different nebulizers and compressors was assessed using constant flow of air from a vacuum pump and a sinusoidal pump to represent typical breathing pat- terns from infancy to the teenage years, directly assaying the amount of drug delivered by the nebulizer.

\section{Materials and methods}

The following nebulizer-compressor combinations were evaluated: Cirrus with Novair II Compressor (Intersurgical, Wokingham, UK), Sidestream with Portaneb 50 compressor (Medicaid, Pagham, UK) and Pari LC Plus with Juniorboy Compressor (Pari Medical, Surrey, UK).

The nebulizers were tested using either a constant flow from a vacuum pump (Edwards ECB8; Edwards, Surrey, $\mathrm{UK}$ ) of 20 or $60 \mathrm{~L} \cdot \mathrm{min}^{-1}$, or a breathing simulator (Sinus Breathing Simulator; Pari, Starnberg, Germany). The experimental apparatus is illustrated in figure 1 .

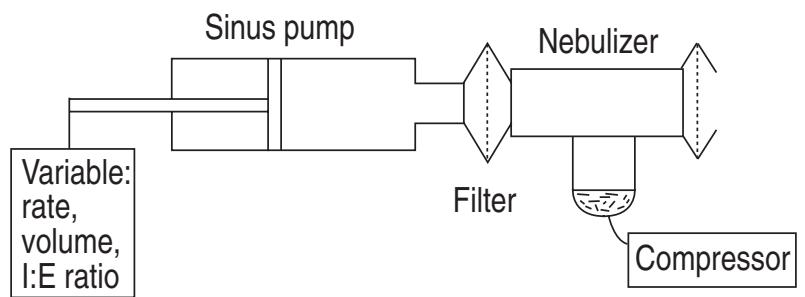

Fig. 1. - Schematic diagram of the experimental apparatus. In the constant-flow experiments, the Edwards' vacuum pump replaced the sinus pump. I:E ratio: inspiratory to expiratory time ratio. 
Electrostatic filter pads were used, held in a plastic filter assembly (dead space $11 \mathrm{~mL}$ ). Nebulizers were connected to the filter assembly by the T-piece (Cirrus and LC Plus) or mouth-piece (Sidestream) supplied by the nebulizer manufacturer. This mouthpiece did not occlude the Sidestream open-vent. Waste aerosol released to the atmosphere during "expiration" was scavenged onto a second filter placed near the end of the T-piece (Cirrus) or entrainment hole of the Sidestream mouthpiece using a household vacuum cleaner. The expiratory filter was connected to the second limb of the T-piece supplied with the LC Plus, and contained a one-way valve so that airflow would be directed through the nebulizer during "inspiration", imitating patient use. The exact arrangement for each nebulizer is illustrated in figure 2 .

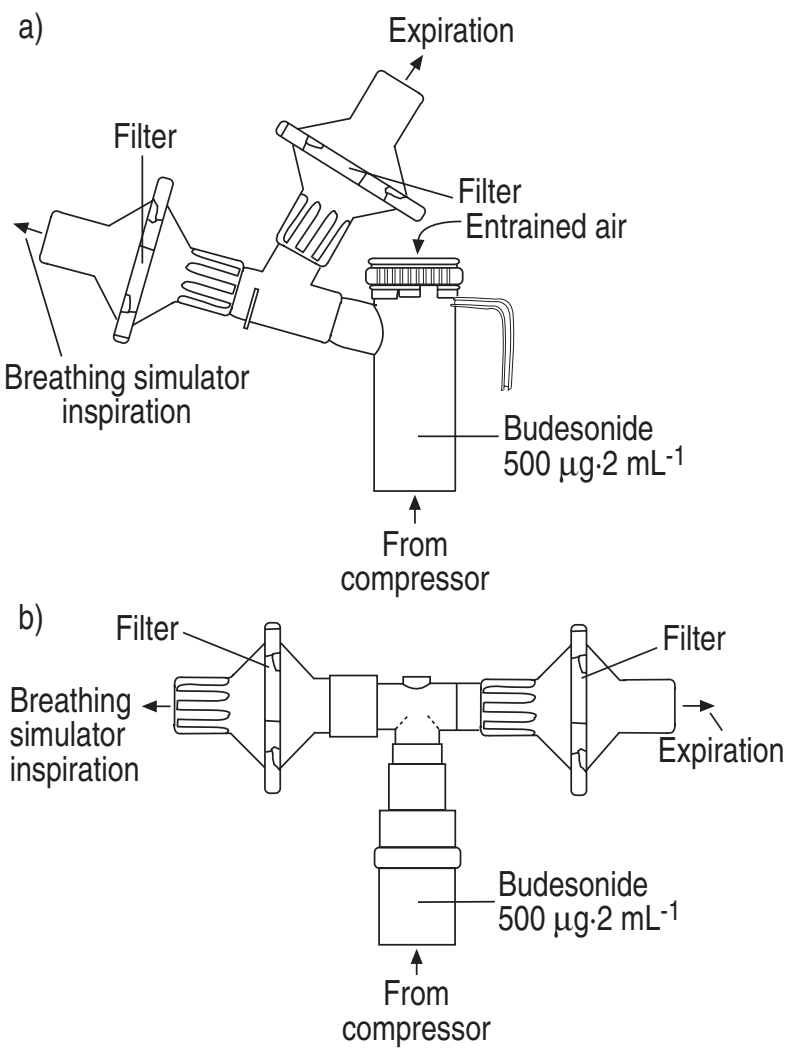

c)

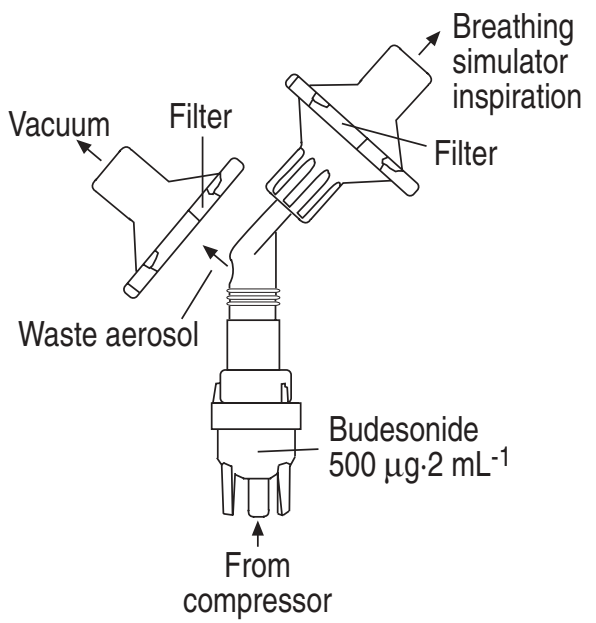

Fig. 2. - Diagram showing the arrangement of nebulizers and filters: a) LC Plus, b) Cirrus, and c) Sidestream.
Table 1. - Breathing simulator settings

\begin{tabular}{lcccccc}
\hline & \multicolumn{5}{c}{ Representative age } \\
\cline { 2 - 7 } & 6 months & 1 yr & 3 yrs & 5 yrs & 10 yrs & 16 yrs \\
\hline$V_{\mathrm{T}} \mathrm{mL}$ & 50 & 75 & 125 & 150 & 225 & 600 \\
$f_{\mathrm{R}}$ breaths $\cdot \mathrm{min}^{-1}$ & 30 & 28 & 24 & 20 & 16 & 16 \\
I:E ratio & $40: 60$ & $40: 60$ & $40: 60$ & $40: 60$ & $40: 60$ & $40: 60$
\end{tabular}

$V \mathrm{~T}$ : tidal volume; $f \mathrm{R}$ : respiratory frequency; I:E ratio: inspiratory:expiratory time ratio.

The respiratory frequency $(f \mathrm{R})$, tidal volume $(V \mathrm{~T})$ and inspiratory to expiratory ratio of the breathing simulator were varied to represent the breathing patterns of children at various ages, as shown in table 1 .

Two millilitres of budesonide suspension (Pulmicort Nebulizer Suspension $250 \mu \mathrm{g} \cdot \mathrm{mL}^{-1}$; Astra, Kings Langley, UK) was added to the nebulizer. This was operated for 5 min, after which the inspiratory filter was removed from the housing and the amount of budesonide deposited on the filter was assayed by high-performance liquid chromatography (HPLC). Each nebulizer was assessed at each breathing pattern and flow a minimum of six times.

The limit of detection of the HPLC assay was $0.1 \mu \mathrm{g}$ budesonide $\cdot \mathrm{mL}^{-1}$. The system response was linear over a range of budesonide concentrations from $0.1-200 \mu \mathrm{g} \cdot \mathrm{mL}^{-1}$. The coefficient of variation of the assay $(n=6)$ was $2.6 \%$ at a concentration of $1 \mu \mathrm{g} \cdot \mathrm{mL}^{-1}$.

In the main experiments, the amount of budesonide on the inspiratory filter only was assayed, but in pilot studies using the same experimental apparatus, the amount of drug deposited in all parts of the experimental apparatus was measured. Of the nominal $500 \mu \mathrm{g}$ budesonide placed in the nebulizer, $94.1 \%$ (95\% confidence intervals (CI) 88.7-99.4) was recovered. Less drug was recovered from the Sidestream nebulizer than from the other nebulizers studied, as some losses occurred from the entrainment ports (fig. 2).

Pilot studies with the filters showed that the recovery of budesonide from primed filters was $100.4 \%$ (95\% CI 97.5-103.3) of $200 \mu \mathrm{g}$ of drug. The filters collected all budesonide particles, with the recovery of budesonide from the second of two filters in line being less than the limit of detection of the assay.

To determine the amount of drug passively collected on the inspiratory filter, the experiments were also undertaken with the breathing simulator switched off (tidal volume $0 \mathrm{~mL}$ ). In the LC Plus, $8 \mu \mathrm{g}$ (95\% CI 6.6-9.4) of budesonide was recovered from the inspiratory filter, compared with only $2.3 \mu \mathrm{g}$ (95\% CI 2.0-2.6) of budesonide with the other nebulizers.

The recovery of budesonide from the inspiratory filter was compared between flows with each nebulizer and between nebulizers at the same flow using analysis of variance (ANOVA). Statistical significance was assumed at $\mathrm{p}=0.05$.

\section{Results}

The amount of budesonide recovered from the filter in the different experiments is given in table 2 . The drug output from all of the nebulizers was greatest when they were assessed under constant flow and was reduced by the use of a simulated breathing pattern. It can be seen from table 
Table 2. - Amount of budesonide recovered from the filters with different nebulizers and breathing patterns

\begin{tabular}{|c|c|c|c|}
\hline \multirow[b]{2}{*}{ Experimental method } & \multicolumn{3}{|c|}{ Nebulizer/compressor } \\
\hline & $\begin{array}{l}\text { Cirrus/Novair II } \\
\mu \mathrm{g}\end{array}$ & $\begin{array}{c}\text { Sidestream/Portaneb } \\
\mu \mathrm{g}\end{array}$ & $\begin{array}{l}\text { Pari LC Plus/Pariboy } \\
\mu \mathrm{g}\end{array}$ \\
\hline Constant flow, $20 \mathrm{~L} \cdot \mathrm{min}^{-1}$ & $\begin{array}{c}102.9 \\
(88.3-117.5)\end{array}$ & $\begin{array}{c}150.4 \\
(130.1-170.8)\end{array}$ & $\begin{array}{c}176.2 \\
(158.5-194.0)\end{array}$ \\
\hline Constant flow, $60 \mathrm{~L} \cdot \mathrm{min}^{-1}$ & $\begin{array}{c}122.7 \\
(106.8-138.5)\end{array}$ & $\begin{array}{c}130.0 \\
(114.4-145.5)\end{array}$ & $\begin{array}{c}93.3 \\
(89.5-97.2)\end{array}$ \\
\hline$V$ T $600 \mathrm{~mL}$ & 35.5 & 19.3 & 82.4 \\
\hline$f \mathrm{R} 16$ breaths $\cdot \mathrm{min}^{-1}$ & $(33.5-37.4)$ & $(14.6-24.1)$ & $(79.0-85.7)$ \\
\hline$V$ T $225 \mathrm{~mL}$ & 39.4 & 17.4 & 70.9 \\
\hline$f \mathrm{R} 16$ breaths $\cdot \min ^{-1}$ & $(33.9-45.0)$ & $(13.0-21.8)$ & $(65.3-76.5)$ \\
\hline$V_{\mathrm{T}} 150 \mathrm{~mL}$ & 34.1 & 18.2 & 73.5 \\
\hline$f \mathrm{R} 20$ breaths $\cdot \min ^{-1}$ & $(28.6-39.7)$ & $(13.1-23.4)$ & $(64.1-82.9)$ \\
\hline$V_{\mathrm{T}} 125 \mathrm{~mL}$ & 37.1 & 18.5 & 69.3 \\
\hline$f \mathrm{R} 24$ breaths $\cdot \mathrm{min}^{-1}$ & $(32.4-41.7)$ & $(17.0-20.0)$ & $(61.1-77.4)$ \\
\hline$V$ T $75 \mathrm{~mL}$ & 27.4 & 13.5 & 71.3 \\
\hline$f_{\mathrm{R}} 28$ breaths $\cdot \mathrm{min}^{-1}$ & $(24.5-30.3)$ & $(12.0-15.0)$ & $(65.7-76.9)$ \\
\hline$V$ T $50 \mathrm{~mL}$ & 4.4 & 4.5 & 25.0 \\
\hline$f \mathrm{R} 30$ breaths $\cdot \mathrm{min}^{-1}$ & $(3.4-5.6)$ & $(3.4-5.6)$ & $(14.1-35.8)$ \\
\hline
\end{tabular}

Values are the mean and 95\% confidence intervals in parentheses for the recovery of budesonide in $\mu \mathrm{g}$. $V \mathrm{~T}$ : tidal volume; $f \mathrm{R}$ : respiratory frequency.

2 that the measured output of the nebulizers may vary by over $700 \%$, depending on the method of measurement.

The output of the LC Plus was markedly reduced at higher flow, from $176 \mu \mathrm{g}$ at $20 \mathrm{~L} \cdot \mathrm{min}^{-1}$ to $93.3 \mu \mathrm{g}$ at 60 $\mathrm{L} \cdot \mathrm{min}^{-1}(\mathrm{p}<0.001)$. The output from the Sidestream was also reduced at the higher flow, and that from the Cirrus increased, but these changes did not reach statistical significance.

Budesonide output was lowest at a tidal volume of 50 $\mathrm{mL}$. The nebulizers all gave constant output at tidal volumes above $125 \mathrm{~mL}$, and at any one breathing pattern the LC Plus deposited more budesonide on the inspiratory filter than the other nebulizers $(\mathrm{p}<0.001)$.

\section{Discussion}

This study has demonstrated significant differences in the amount of drug produced by nebulizers depending on the exact method of testing. In the past, nebulizers have been tested by weighing before and after operation for a certain time, without any additional airflow to represent inhalation by the patient [4]. This method does not take into account the evaporation of water during nebulization and overestimates drug output [5]. To overcome this, a recent study [6] has proposed the use of a tracer (sodium fluoride) in the nebulizer solution, which is captured on a filter and assayed. However, a constant flow of air is used to draw aerosol onto the filter which, as shown in this study, will lead to an inaccurate measurement of the drug output. A number of comparative studies has measured drug output from nebulizers under constant or no entraining flow [7-9]. The present data suggest that, for some nebulizers, these methods may give very misleading results.

The recently published British Standard Institute's specification for nebulizers [1], which specifies the information about nebulizers that should be provided by manufacturers and the test methodologies that should be employed, also ignores the importance of simulated breathing pattern. This should be taken into account when European standards are formulated.
Nebulized drug deposition in the lungs is affected by the size of drug particles produced. This study measured the total amount of drug released from the nebulizer and deposited on the inspiratory filter, without measuring particle size, and therefore the results cannot be used to suggest drug delivery to the lungs. Aerosol particle size may be measured by inertial impaction devices, which commonly sample aerosol at a high flow rate. For instance, an Andersen Impactor samples at $28.3 \mathrm{~L} \cdot \mathrm{min}^{-1}$, whereas a multistage liquid impinger samples at $60 \mathrm{~L} \cdot \mathrm{min}^{-1}$. The results suggest that connecting a Pari LC Plus nebulizer to these two devices would give very different results. Assessing nebulizers at high flows may be detrimental, presumably because the high flow through the nebulizer encourages impaction of particles on the internal baffles. Breath-enhanced, open-vent nebulizers such as the LC Plus and Ventstream nebulizers (Medicaid, Pagham, UK) are designed to use the patient's inspiratory flow to increase nebulizer output [10] and particle size may also change with different flows through these nebulizers. Clearly, assessing these nebulizers under constant flow conditions will not reliably measure their output during use by patients.

Many factors, such as driving gas flow rate and nebulizer design, will determine the output of drug from a nebulizer [11]. The actual dose reaching the lungs of the patient will also be influenced by the anatomy of the upper airway, the degree of airway obstruction and the breathing pattern of the patient. Others have demonstrated the effect of entrained air diluting the inspired aerosol $[2,12]$, so that, from conventional nebulizers, the amount of drug inhaled is independent of tidal volume once inspiratory flow is greater than the nebulizer driving gas flow. The results for the Cirrus nebulizer, where deposition on the filter is constant at tidal volumes above $125 \mathrm{~mL}$, support this. The output from this nebulizer when assessed with the breathing simulator was just under $40 \%$ of that recovered under constant flow, which would have been predicted from the use of an inspiratory fraction of $40 \%$ of the breathing cycle. 
The Sidestream nebulizer is supplied with a mouthpiece containing an entrainment hole. During the breathing simulation experiments, aerosol was seen to escape from this hole throughout the breathing cycle (fig. 2). The very low deposition of drug on the inspiratory filter may be due to the enhanced output of this nebulizer being greater than the inspiratory flow, so that aerosol is wasted during both inspiration and expiration. The nebulizer produces small particles, since large droplets are filtered out by baffles in the nebulizer. As budesonide is a suspension of drug particles of approximately 2.2-2.9 $\mu \mathrm{m}$ median diameter [13], many drug particles will be too large to be released from the nebulizer.

Breath-enhanced, open-vent nebulizers increase drug output during inspiration by using the inspiratory flow to open a valve through which air is entrained [11]. As airflow increases, particles that would have remained in the nebulizer are reduced in size by greater evaporation and are released to the patient. This increasing output is counteracted above a certain flow by increased impaction within the nebulizer, resulting in a constant and, finally, a reduced drug output [14].

This in vitro study has demonstrated the importance of using simulated breathing patterns in the assessment of nebulizers. However, a sinus wave form was used to imitate the required breathing patterns and this may not be representative of real patient's breathing. DENYER et al. [15] suggested that derived sinus wave forms based on real patients' breathing rate and tidal volume, parameters such as peak inspiratory flow may be incorrect by more than $35 \%$. Breathing frequencies and tidal volumes may also change when patients have a tightly fitting mask placed over their mouth and nose, compared with their breathing pattern at rest. It is unclear how much difference this makes to measured drug output and more sophisticated breathing simulators are awaited. When these are available, regulatory authorities and nebulizer manufacturers will need to define representative breathing patterns for comparative testing of devices.

The present study has demonstrated a pitfall of current nebulizer testing standards that will need to be corrected if future standards are to give meaningful results for the testing of nebulizers.

Acknowledgements: The authors would like to thank B. Morjaria for technical support, Medical Illustration, Leicester Royal Infirmary, for drawing figure 2, the various nebulizer companies who supplied the nebulizers and compressors and Pari Medical for supplying the breathing simulator.

\section{References}

1. British Standards Institute. BS7711 Part 3. Specification for gas powered nebulisers for the delivery of drugs, London, British Standards Institute, 1994.

2. Collis GG, Cole CH, Le Souef PN. Dilution of nebulised aerosols by air entrainment in children. Lancet 1990; 336: 341-343.

3. Newnham DM, Lipworth BJ. Nebuliser performance, pharmacokinetics, airways and systemic effects of salbutamol given via a novel nebuliser delivery system ("Ventstream"). Thorax 1994; 49: 762-770.

4. Kradjan WA, Lakshminarayan S. Efficiency of air compressor driven nebulisers. Chest 1985; 87: 512-516.

5. O'Callaghan C, Clarke AR, Milner AD. Inaccurate calculation of drug output from nebulisers. Eur J Pediatr 1989; 148: 473-474.

6. Dennis JH, Stenton SC, Beach JR, Avery AJ, Walters EH, Hendrick DJ. Jet and ultrasonic nebuliser output: use of a new method for direct measurement of aerosol output. Thorax 1990; 45: 728-732.

7. Smith EC, Denyer J, Kendrick AH. Comparison of twenty three nebulizer/compressor combinations for domiciliary use. Eur Respir J 1995; 8: 1214-1221.

8. Hurley PK, Smye SW. Performance assessment of a range of commercial jet nebulisers. Technol Health Care 1994; 1: 209-214.

9. Hung JCC, Hambleton G, Super M. Evaluation of two commercial jet nebulisers and three compressors for the nebulisation of antibiotics. Arch Dis Child 1994; 71: 335338.

10. Nikander K. Drug delivery systems. J Aerosol Med 1994; (Suppl.) 7: S19-S23.

11. O'Callaghan C, Barry PW. The science of nebulised drug delivery. Thorax 1997; 52 (Suppl. 2): S31-44.

12. Everard ML, Clark AR, Milner AD. Drug delivery from jet nebulisers. Arch Dis Child 1992; 67: 586-591.

13. Jackson WF. Nebulised budesonide therapy in asthma. Harwell, Clinical Vision, 1995; p. 40.

14. Knoch M, Wunderlich E. In vitro assessment of a new efficient nebuliser system for continuous operation. In: Conference Proceedings, Respiratory Drug Delivery IV, Richmond, VA, Virginia Commonwealth University, 1994.

15. Denyer J, Dyche A, Nikander K. Breathing patterns in adult patients. In: Conference Proceedings, ISAM Focus Symposium, Tours, France, International Society of Aerosols in Medicine, 1996. 